Cerezo Ramírez, F. \& Rubio Hernández, F.J. (2017). Medidas relativas al acoso escolar y ciberacoso en la normativa autonómica española. Un estudio comparativo. Revista Electrónica Interuniversitaria de Formación del Profesorado, 20(1), 113-126.

\title{
Medidas relativas al acoso escolar y ciberacoso en la normativa autonómica española. Un estudio comparativo
}

Fuensanta Cerezo Ramírez, Francisco José Rubio Hernández.

Universidad de Murcia

\section{Resumen}

Sendos informes del Defensor del Pueblo Español alertaron de la necesidad de adoptar medidas para controlar el acoso entre escolares y mejorar la convivencia en las aulas. Sus recomendaciones fueron recogidas por las Comunidades Autónomas (CC.AA.) y plasmadas en normativas propias. El presente artículo reflexiona sobre las medidas adoptadas, como normas de convivencia, por las administraciones públicas, para los centros educativos sostenidos con fondos públicos. Se plantea un estudio comparativo sobre la normativa relacionada con los fenómenos de acoso escolar o bullying y ciberacoso o cyberbullying en ocho CC.AA. seleccionadas al azar. El objetivo principal es conocer cómo se ha abordado esta problemática, así como comparar los protocolos de actuación propuestos y el carácter de las medidas recogidas para su intervención en cada CC.AA. Los resultados indican que la mayoría de las políticas hacen referencia expresa al bullying y una de cada cuatro menciona el cyberbullying, también se detectó que la mitad de los protocolos proponen medidas de actuación ante violencia escolar. En cuanto al carácter de las medidas adoptadas, el análisis demuestra que éstas se dirigen, casi exclusivamente, al alumnado agresor y que, en la mayoría de los casos, son de índole correctiva/disciplinaria. Se destaca que en las normativas apenas se hace referencia a medidas de carácter educativo, dejando sin respuesta la propuesta del Defensor del Pueblo que hacía hincapié en las medidas educativas.

\section{Palabras clave}

Acoso escolar; ciberacoso; políticas educativas; violencia escolar. 


\title{
Policies against Bullying and Cyberbullying in Spanish autonomic laws. A comparative study
}

\begin{abstract}
Two reports of the Spanish Ombudsman warn of the need to adopt measures to control bullying among schoolchildren and improve coexistence in the classroom. Their recommendations were taken up by the Autonomous Communities (AA.CC.) and reflected in their own regulations. This article reflects on policies taken as standards of coexistence by public government, for schools supported with public funds. A comparative study was carried out on the regulations related to bullying and cyberbullying in eight Spanish regions, randomly selected. The main objective is to know how this issue is addressed and compare protocols proposed action and the nature of the intervention measures provided for in each AA.CC. Results show that most policies specifically refer to bullying, and one in four mentioned cyberbullying, also found that half of the protocols proposed measures performance against school violence. As for the nature of the measures, the analysis shows that they are directed, almost exclusively, to the assailant students and, in most cases, are corrective / disciplinary nature. Among the findings it highlighted that every policy scarcely indicated educational measures, leaving unanswered the proposal of the Ombudsman that emphasized that educational measures.
\end{abstract}

\section{Key words}

Cyberbullying; violence prevention; educative policies; school violence.

\section{Introducción}

Los estudios sobre acoso escolar comienzan en la década de los años sesenta, momento en el que algunos investigadores detectan como motivo detonante del suicidio de adolescentes el acoso escolar que estos sufren. No obstante, es en 1978 cuando el psicólogo escandinavo Dan Olweus introduce el término bullying, con el que es conocido este fenómeno internacionalmente. De esta forma, en la actualidad existe un amplio consenso en reconocer que las personas agredidas sufren el bullying como "una auténtica pesadilla", cuyas secuelas alcanzan tal magnitud que, en ocasiones, tienen prevalencia, incluso, en su vida adulta, lo que cual ha hecho promover políticas de prevención, evaluación e intervención en diferentes estados. (Prodócimo, Cerezo, \& Arense, 2014)

Por otro lado, la definición convencional de bullying incluye tres características: intencionalidad, desequilibrio de poder entre agresor y víctima y la repetición de la conducta en el tiempo (Olweus, 1996, 1998; Solberg \& Olweus, 2003). Por tanto, entendemos por acoso escolar toda agresión física o psicológica de un escolar o grupo de escolares hacia otro, frecuente, que se mantiene en el tiempo, y representa un exponente de las malas relaciones entre escolares (Ortega, Sánchez, \& Menesini, 2002) unido a un deseo perverso de diversión (Cerezo, 2009b). 
Asimismo, otro aspecto a destacar del acoso entre escolares es su carácter social, ya que los resultados de estudios sobre este fenómeno apuntan algunas características socio-afectivas sobre los escolares implicados en la dinámica bullying e indican que estos escolares presentan una posición social desfavorable respecto a los que no están implicados (GarcíaBacete, Sureda, \& Monjas, 2010). Este aspecto señala la necesidad de conocer las percepciones y actitudes del profesorado (Benítez, Berbén, \& Fernández, 2006), así como de la estructura socio-afectiva del grupo como mecanismo subyacente que contribuye a mantener la relación agresión-victimización, siendo por tanto su análisis necesario para orientar las actuaciones preventivas encaminadas a mejorar la convivencia escolar (Cerezo, 2014).

De forma paralela al bullying presencial, asistimos desde hace unos años al exordio de cambios en la realidad de la violencia, dando lugar a nuevas estructuras de agresión entre iguales, como es el caso del ciberacoso, o cyberbullying (Caballo, Calderero, Arias, Salazar, \& Irurtia, 2012; Simth, Mahdavi, Carvallo, Fisher, Russel, \& Tippet, 2008). En este tipo de acoso se utilizan las Tecnologías de la Información y la Comunicación (TIC), principalmente Internet y el teléfono móvil (Calvete, Orue, Estévez, Villardón, \& Padilla, 2010; Garaigordobil \& Aliri, 2013; Durán-Segura \& Martínez-Pecino, 2015). El cyberbullying traspasa el espacio y el tiempo (Cerezo, 2012), facilita el ataque desde el anonimato y en espacios virtuales, a los cuales cualquiera tiene acceso (Cerezo, Arnaiz, Giménez, \& Maquilón, 2016), lo cual lo convierten en un fenómeno con una alta prevalencia (Garaigordobil, 2011). En este sentido, la falta de control parental, las conductas poco seguras en Internet, el uso de redes sociales, así como los programas de mensajería instantánea y la frecuencia de uso de Internet durante el fin de semana son factores de riesgo tanto de cibervictimización ocasional como habitual (Álvarez-García, Núñez, Dobarro, \& Rodríguez, 2015).

Además, tanto el bullying como el cyberbullying se consideran factores de riesgo social y emocional asociado a problemas psicológicos (Cerezo 2009a; Moreno, Estévez, Murgui, \& Musitu, 2009; Musitu, Estévez, Jiménez, \& Veiga, 2011), que trascienden a la vida escolar, constituyendo un claro antecedente de las conductas disociales (Bender y Lösel, 2011). Así, se encuentran comportamientos relacionados con cyberbullying entre adolescentes, tales como la recepción de amenazas a través de correo electrónico, de insultos a través de la red y también emitir insultos a otros compañeros mediante las diferentes aplicaciones presentes en Internet (Fernández-Montalvo \& Peñalva, 2015; Horner, Asher, \& Fireman, 2015). No obstante, las TIC constituyen a su vez instrumentos convenientes para intervenciones con niños y adolescentes que han sufrido estos fenómenos, ya que promueven su bienestar emocional, psicológico y social (Nocentini, Zambuto, \& Menesini, 2015).

En otro orden de cosas, el Informe de Save the Children (2016) recoge que la totalidad de niños y niñas tienen derecho a ser protegidos de todas las formas de violencia y a posibilitarles el desarrollo de todo su potencial de aprendizaje en un ambiente seguro. Propuesta que ya contempla la Convención de Naciones Unidas sobre los Derechos del Niño (1989) , y desarrolla ampliamente el Comité de Derechos del Niño (2011b), como el Comité de los Derechos Económicos, Sociales y Culturales (2011a), haciendo mención explícita al cyberbullying. A pesar de este panorama favorable, las leyes y políticas sobre bullying aún se encuentran fragmentadas y son inconsistentes (Comer \& Limber, 2015). La actuación preventiva y correctora ha de ser un objetivo primordial para los centros escolares, dado que estas instituciones tienen un papel fundamental en la protección de la infancia contra cualquier tipo de violencia (Ortega y Núñez, 2012). Sin embargo, las aulas son en ocasiones escenarios de exclusión social y maltrato que demandan una intervención planificada. Así, previo a cualquier intervención es preciso llevar a cabo una evaluación individualizada que 
permita establecer las medidas sancionadoras y educativas adecuadas, siempre respetando los derechos que reconoce la Convención (1989), que defiende la sustitución de la represión y el castigo, por medidas de índole rehabilitadora y restitutiva (Naciones Unidas, 2011b). Además, al tratarse de un fenómeno grupal (Ortega, 2008), se deben tomar medidas hacia el grupo de iguales.

El contexto normativo español de forma genérica, establece una cobertura legal en relación al acoso en diferentes documentos, así, la Constitución Española de 1978 y el preámbulo de la Ley de Ordenación General del Sistema Educativo (LOGSE, 1990). En el Real Decreto 732/1995, de 5 de mayo, se establecen los Derechos y Deberes de los alumnos y las normas de convivencia en los centros educativos y se señala el carácter educativo y recuperador de las sanciones. Asimismo, la Ley Orgánica de Educación de 2/2006 reconoce el importante papel de la institución escolar en la prevención y lucha contra el bullying, y para la educación en la no violencia. En este sentido, cabe señalar que, según las recomendaciones de sendos informes del Defensor del Pueblo, (AA.VV. 2000; AA.VV. 2007), es necesaria una mayor implicación de los diferentes sectores sociales en la prevención e intervención del acoso escolar. Con esta finalidad se creó el Observatorio de la Violencia Escolar, nacional y en las distintas CCAA., y se indica que todos los centros escolares deben elaborar el denominado Plan de Convivencia. Además, como resultado de la nueva consideración social y cultual del bullying, la legislación educativa incluye una referencia a las víctimas de acoso escolar en la Ley Orgánica de Educación (LOE, 2006). No obstante, podemos afirmar que no existe en nuestro país una ley que regule de forma específica e integral este problema.

Aunque en el Código Penal (Ley Orgánica 10/1995, de 23 de noviembre, del Código Penal) no existe alusión expresa al acoso escolar, sí se señala implícitamente que quien inflija un trato degradante que menoscabe gravemente la integridad moral, será castigado con la pena de prisión de seis meses a dos años, pero el alumnado, agente de las agresiones escolares, es un sujeto menor de edad, por lo que no es posible aplicarle la consecuencia al no cumplimiento de esta norma (Fanjul, 2012). En todo caso puede acogerse a una responsabilidad penal específica y no ordinaria, como recoge la Ley Orgánica reguladora de la Responsabilidad Penal del Menor (LORPM, 2006).

Incluso la propia fiscalía general del Estado Español, en su instrucción 10/2005, alerta de la aceptación pasiva del acoso escolar como si fuese algo inherente a la vida de los centros escolares, por lo que ahonda en la propuesta para sancionar estos hechos y eleva a la categoría de falta o delito esta problemática social. Así pues nos encontramos ante la necesidad de contar con un marco legislativo que aborde el bullying y el cyberbullying, agresiones que, desde la generalización del uso de las TIC, transfieren los escenarios físicosComo respuesta a estas indicaciones algunas comunidades autónomas generaron sus propias normativas, por lo que hace más de una década ya contamos con políticas educativas que contemplan una intervención específica, aunque diferente en cada comunidad. Algunas de las diecisiete Comunidades Autónomas españolas, van mas allá y crean órganos de control y establecen instrumentos normativos, recogidos en leyes, decretos, órdenes y/o resoluciones para hacer frente al acoso escolar (Cerezo, 2011). Del mismo modo, se elaboran los Planes de Convivencia, que constituyen el marco de referencia para la gestión del bullying y del cyberbullying, regulando procesos y elaborando protocolos a seguir (Viana-Orta, 2013). Nos encontramos que, después de más de una década de estas iniciativas, no conocemos su alcance real ni su ajuste a las indicaciones propuestas por los diferentes expertos.

Teniendo en cuenta todo lo anterior, consideramos pertinente y necesario el análisis de la normativa autonómica española referente a estos fenómenos -acoso escolar y ciberacoso-, tan actual como preocupantes, ya que deben de contar con un respaldo legal y preceptivo 
que atienda tanto a las consecuencias del fenómeno como a sus protagonistas. Este trabajo pretende poner de manifiesto el tratamiento legal y las medidas propuestas por las diferentes CCAA para el tratamiento de la violencia escolar, comprobando su nivel de ajuste a las demandas educativas necesarias, así como el grado de inclusión de medidas específicas ante el bullying y cyberbullying.

Para el estudio se han seleccionado ocho CCAA.

\section{Objetivos e hipótesis}

El objetivo general de este trabajo es describir y comparar cómo se atiende y responde desde la normativa educativa de ocho comunidades autónomas españolas al acoso escolar y ciberacoso.

Este objetivo general se concreta en los siguientes objetivos específicos:

1. Detectar si se incluye expresamente los términos bullying y/o cyberbullying en la normativa de cada una de las ocho comunidades autónomas seleccionadas.

2. Indagar si la normativa de cada una de las ocho comunidades autónomas seleccionadas propone y/o recoge protocolos de prevención, detección y actuación/intervención relacionados con el bullying y cyberbullying.

3. Analizar el carácter de las medidas (educativo, restitutivo y/o correctivo) de la normativa de cada una de las ocho comunidades autónomas seleccionadas.

4. Localizar y describir las coincidencias y diferencias respecto a la atención y respuesta que se da a los problemas de acoso escolar y ciberacoso en la normativa de cada una de las ocho comunidades autónomas seleccionadas.

5. Describir el grado de respaldo normativo que están recibiendo por parte de las ocho comunidades autónomas seleccionadas los fenómenos de bullying y cyberbullying.

Se establecen las siguientes hipótesis:

1. El término acoso escolar estará incluido expresamente en las normativas educativas.

2. El término ciberacoso estará incluido expresamente en las normativas educativas.

3. Las distintas normativas proponen o anexan protocolos de prevención, detección y actuación/intervención relacionados con el bullying.

4. Las distintas normativas proponen o anexan protocolos de prevención, detección y actuación/intervención relacionados con el cyberbullying.

5. Las leyes, decretos, órdenes y resoluciones recogidas y publicadas durante el período de tiempo comprendido entre los años 2009 y 2015 conceden mayor respaldo normativo al fenómeno del bullying que las de años anteriores.

\section{Metodología}

Se trata de un estudio comparativo (Raventós, 1983; Velloso, 2005), método propio de la Educación Comparada. Para ello, se realizó una revisión sistemática o meta-análisis (Sánchez-Meca y Botella, 2010) de los documentos normativos de las ocho comunidades 
autónomas respecto al bullying y cyberbullying. Partiendo de la búsqueda de legislación autonómica en diciembre de 2015 en la página web del Boletín Oficial del Estado, así como de los boletines oficiales de las distintas comunidades autónomas objeto de estudio (Andalucía, Canarias, Castilla y León, Cataluña, Galicia, Madrid, Murcia y País Vasco). Los descriptores utilizados fueron: "convivencia escolar", "acoso escolar" y "ciberacoso", y los resultados de búsqueda de 15 registros obtenidos (véase Tabla 1), atendiendo a los siguientes criterios o limitaciones:

- Tipo de documento: legislación autonómica.

- Carácter de las disposiciones: vigente.

- Departamento: Comunidades autónomas de Andalucía, Canarias, Castilla y León, Cataluña, Galicia, Madrid, Murcia y País Vasco.

Tras esto, se ejecutaron las tres fases del método comparativo (descripción, yuxtaposición y comparación).

En primer lugar se procedió, en la etapa de descripción, al análisis y síntesis de los aspectos relacionados con el acoso escolar y ciberacoso presentes en la normativa educativa de cada una de las comunidades autónomas seleccionadas. Dichos aspectos fueron: presencia explícita del término "acoso escolar" y/o "ciberacoso", carácter de las medidas contempladas para el agresor y la víctima, y la anexión o no de protocolos de intervención.

En segundo lugar, se confrontaron, punto por punto, los datos recogidos en la fase de descripción.

Finalmente, se compararon las informaciones y examinaron las diferencias y semejanzas respecto al respaldo y respuesta que cada una de las comunidades autónomas seleccionadas daban al acoso escolar y ciberacoso en sus normativas.

Las unidades comparativas del estudio fueron ocho $(n=8)$ de las diecisiete comunidades autónomas españolas: Andalucía, Canarias, Castilla y León, Cataluña, Galicia, Madrid, Murcia y País Vasco).

Los criterios de inclusión de las comunidades autónomas se caracterizaron por estar situada en unos de los cuadrantes que resultaron de subdividir la totalidad del mapa autonómico en cuatro sectores: noreste; noroeste; sureste; suroeste. Se seleccionaron aleatoriamente dos comunidades de cada sector.

Las variables fueron establecidas según los puntos de la normativa educativa (leyes, decretos, órdenes y resoluciones) relacionada con el acoso escolar y ciberacoso, en concreto se consideraron:

1. Inclusión del término Acoso escolar, o bullying.

2. Inclusión del término ciberacoso o cyberbullying.

3. Carácter de las medidas adoptadas: educativo, restitutivo (reintegrador o recuperativo) y/o correctivo.

Los documentos normativos (leyes, decretos, órdenes y resoluciones) fueron aquellos relativos al ámbito educativo y publicados por las comunidades autónomas seleccionadas (véase Tabla 1). 
Tabla 1.

Normativa educativa autonómica revisada

\begin{tabular}{|c|c|}
\hline Comunidad autónoma & Documentos normativos revisados \\
\hline Andalucía & $\begin{array}{l}\text { Decreto } 19 / 2007 \text {, de } 23 \text { de enero. } \\
\text { Orden de } 20 \text { de junio de } 2011 .\end{array}$ \\
\hline Canarias & Decreto 114/2011, de 11 de mayo. \\
\hline Castilla y León & $\begin{array}{l}\text { Orden EDU/52/2005, de } 26 \text { de enero. } \\
\text { Decreto 51/2007, de } 17 \text { de mayo. } \\
\text { Orden EDU/1921/2007, de } 27 \text { de noviembre. }\end{array}$ \\
\hline Cataluña & $\begin{array}{l}\text { Decreto } 279 / 2006 \text {, de } 4 \text { de julio. } \\
\text { Ley } 12 / 2009 \text {, de } 10 \text { de julio. }\end{array}$ \\
\hline Galicia & $\begin{array}{l}\text { Ley } 4 / 2011 \text {, de } 30 \text { de junio. } \\
\text { Decreto } 8 / 2015 \text {, de } 8 \text { de enero. }\end{array}$ \\
\hline Madrid & Decreto $15 / 2007$, de 19 de abril. \\
\hline País Vasco & $\begin{array}{l}\text { Decreto n. }{ }^{\circ} 119 / 2005 \text {, de } 21 \text { de octubre. } \\
\text { Resolución de } 4 \text { de abril de } 2006 . \\
\text { Resolución de } 28 \text { de septiembre de } 2009 \text {. } \\
\text { Decreto } 201 / 2008 \text {, de } 2 \text { de noviembre. }\end{array}$ \\
\hline
\end{tabular}

\section{Resultados}

Se presentan los resultados en el mismo orden que los objetivos e hipótesis, cuyos principales datos de recogen en la Tabla 2.

En relación al objetivo específico 1, los resultados confirmaron que el $87,5 \%$ de la normativa educativa de las comunidades autónomas $(n=7)$ incluyó expresamente el término acoso escolar. Únicamente la Comunidad de Madrid $(n=1)$ no explicitó ni abordó el bullying en sus documentos normativos. De esta forma, se rechazó la hipótesis 1, porque no se recogió de forma explícita en toda la normativa educativa autonómica comparada el término acoso escolar.

Además, solo el $25 \%$ de las CCAA (Andalucía y Galicia) recogían en su normativa de forma expresa el término ciberacoso, por lo que se rechazó la hipótesis de investigación 2, puesto que en la totalidad de la normativa educativa autonómica comparada no se encontró de forma explícita el término cyberbullying.

\section{Tabla 2.}

Síntesis de las fases de descripción y yuxtaposición

Nota: Protocolo tipo A (para bullying). Protocolo tipo B (relativo bullying $y$ cyberbullying) 


\begin{tabular}{|c|c|c|c|c|c|}
\hline $\begin{array}{l}\text { Comunidad } \\
\text { autónoma } \\
\text { seleccionada }\end{array}$ & $\begin{array}{l}\text { Documentos } \\
\text { normativos } \\
\text { Revisados }\end{array}$ & $\begin{array}{l}\text { Presencia } \\
\text { expresa } \\
\text { bullying }\end{array}$ & $\begin{array}{l}\text { Presencia } \\
\text { expresa } \\
\text { cyberbullying }\end{array}$ & $\begin{array}{l}\text { Carácter } \\
\text { de las } \\
\text { medidas }\end{array}$ & $\begin{array}{l}\text { Protocolos } \\
\text { tipos } \\
\text { A o B }\end{array}$ \\
\hline Andalucía & $\begin{array}{l}\text { Orden de } 20 \text { de } \\
\text { junio de } 2011\end{array}$ & Sí & Sí & $\begin{array}{l}\text { Agresor: } \\
\text { Correctivo/ } \\
\text { disciplinario } \\
\text { Víctima: } \\
\text { De apoyo y } \\
\text { protección }\end{array}$ & Protocolo tipo B \\
\hline Canarias & $\begin{array}{l}\text { Decreto } 114 / 2011 \text {, } \\
\text { de } 11 \text { de mayo }\end{array}$ & Sí & No & $\begin{array}{l}\text { Agresor: } \\
\text { Correctivo/ } \\
\text { disciplinario } \\
\text { Víctima: } \\
\text { No contempla }\end{array}$ & No \\
\hline Castilla y León & $\begin{array}{l}\text { Orden } \\
\text { EDU/52/2005, de } \\
26 \text { de enero }\end{array}$ & Sí & No & $\begin{array}{l}\text { Agresor: } \\
\text { Correctivo/ } \\
\text { disciplinario } \\
\text { Víctima: } \\
\text { De apoyo y } \\
\text { protección }\end{array}$ & Protocolo tipo A \\
\hline Cataluña & $\begin{array}{l}\text { Ley } 12 / 2009 \text {, de } \\
10 \text { de julio }\end{array}$ & Sí & No & $\begin{array}{l}\text { Agresor: } \\
\text { Correctivo/ } \\
\text { disciplinario } \\
\text { Víctima: } \\
\text { No contempla }\end{array}$ & No \\
\hline Galicia & $\begin{array}{l}\text { Decreto } 8 / 2015 \text {, } \\
\text { de } 8 \text { de enero }\end{array}$ & Sí & Sí & $\begin{array}{l}\text { Agresor: } \\
\text { Correctivo/ } \\
\text { disciplinario } \\
\text { Víctima: } \\
\text { De apoyo y } \\
\text { protección }\end{array}$ & Protocolo tipo B \\
\hline Madrid & $\begin{array}{l}\text { Decreto } 15 / 2007 \text {, } \\
\text { de } 19 \text { de abril }\end{array}$ & No & No & $\begin{array}{l}\text { Agresor: } \\
\text { No contempla } \\
\text { Víctima: } \\
\text { No } \\
\text { contempla }\end{array}$ & No \\
\hline Murcia & $\begin{array}{l}\text { Resolución de } 4 \\
\text { de abril de } 2006\end{array}$ & Sí & No & $\begin{array}{l}\text { Agresor: } \\
\text { Correctivo/ } \\
\text { disciplinario } \\
\text { Víctima: } \\
\text { De apoyo y } \\
\text { protección }\end{array}$ & Protocolo tipo $\mathrm{A}$ \\
\hline País Vasco & $\begin{array}{l}\text { Decreto } \\
201 / 2008 \text {, de } 2 \\
\text { de noviembre }\end{array}$ & Sí & No & $\begin{array}{l}\text { Agresor: } \\
\text { Correctivo/ } \\
\text { disciplinario } \\
\text { Víctima: } \\
\text { De apoyo y } \\
\text { protección }\end{array}$ & No \\
\hline
\end{tabular}


En cuanto al objetivo específico 2, el $50 \%$ de las comunidades autónomas proponían protocolos específicos, de las que el $25 \%$ fueron relativos al acoso escolar (Castilla y León, y Murcia) y otro $25 \%$ lo hicieron respecto al bullying y al cyberbullying (Andalucía y Galicia)

Por ende, se rechazaron las hipótesis de investigación 3 y 4 , puesto que no en toda la normativa autonómica comparada se anexó o propuso un protocolo de acoso escolar y ciberacoso que abordara la prevención, detección y actuación o intervención del problema.

En el análisis de los textos se hallaron medidas de diversa índole, de las cuales prevalecían las de carácter correctivo y disciplinario, seguidas de las educativas, y, finalmente, las restitutivas respecto a los agresores en situaciones de bullying. Concretamente, el $87,5 \%$ de las comunidades autónomas recogieron medidas de carácter correctivo y/o disciplinario en relación a los acosadores. No se encontraron medidas de carácter educativo.

En cuanto al carácter de las medidas respecto a la víctima, se detectó que el $62,5 \%$ de comunidades autónomas recogían medidas de apoyo y protección para el agredido, frente al $37,5 \%$ no proponían ninguna

Respecto a la hipótesis de investigación 5, se aceptó, ya que las leyes, decretos, órdenes y resoluciones comparadas y publicadas durante el período de tiempo comprendido entre los años 2009 y 2015 concedían mayor respaldo normativo al fenómeno del bullying. Así, de las ocho CCAA que se compararon el $50 \%(n=4)$ publicaron los documentos normativos durante el período 2009-2015, mientras que el 37,5\% hicieron lo propio durante los años 2005 y 2008. El 12,5\% restante, que corresponde a la Comunidad de Madrid, no proclamó normativa específica relativa al acoso escolar hasta el momento.

En otro orden de cosas, en relación a los objetivos específicos 4 y 5 , se concluyó que la principal similitud entre la normativa comparada fue que la mayoría de las comunidades autónomas seleccionadas, es decir, un 87,5\%, $\mathrm{n}=7$ (Andalucía, Canarias, Castilla y León, Cataluña, Galicia, Murcia y País Vasco) abordaban los fenómenos de acoso escolar. Por el contrario, solo dos de ellas, Andalucía y Galicia (25\%), hicieron lo propio con el ciberacoso.

Además, la tendencia general fue que las medidas adoptadas para esta problemática poseían un carácter correctivo/disciplinario, en el caso del agresor o agresora (87,5\%), y de apoyo y protección, en el caso de la víctima (62,5\%). De igual forma, aunque en la mayoría de documentos normativos consultados se recogía la naturaleza educativa de los objetivos y finalidades de las medidas a adoptar en situaciones de acoso escolar en cuanto al sujeto agresor, casi la totalidad de las que se contemplaban en las legislaciones autonómicas aludieron a la disciplina y la corrección, por lo que se dio una contradicción que se tuvo en cuenta.

Entre las diferencias, destacaron las encontradas en la anexión de protocolos dentro del documento normativo de cada una de las comunidades autónomas. Aunque cuatro de ellas (50\%) proponían o recogían uno de estos, no lo hacían de una forma completa. Tampoco tenían en cuenta a todos los miembros de la institución escolar (alumnado, profesorado, familias, etcétera).

\section{Discusión y conclusiones}

El objetivo principal de este estudio fue comprobar cómo abordaban y respondían las diferentes normativas autonómicas a la necesidad de afrontar la violencia escolar, tal y como propuso el Defensor del Pueblo (AA.VV, 2007). El análisis llevado a cabo sobre los documentos de ocho comunidades autónomas refleja que el respaldo normativo es todavía escaso y está poco ajustado a las indicaciones tanto del Defensor del Pueblo como a las 
aportadas por los principales investigadores sobre la temática en nuestro país (Cerezo, 2014; Ortega \& Núñez, 2012; Viana-Orta, 2013), por lo que sigue siendo imprescindible la creación de legislaciones específicas relativas a los fenómenos de bullying y cyberbullying (Save the Children, 2016). Cabe señalar que solo la Región de Murcia cuenta con un documento normativo expreso para esta problemática.

En relación a la inclusión de los términos "acoso escolar" y "ciberacoso", encontramos que todavía son escasas las respuestas normativas que incluyen la forma cibernética de acoso (Garaigordobil \& Aliri, 2013), siendo las TIC una herramienta utilizada con carácter general por la mayoría de nuestros escolares (Fernández-Montalvo \& Peñalva, 2015).

Este trabajo muestra la escasa inclusión de protocolos de actuación específicos en las normativas que regulan la convivencia en los centros escolares mantenidos con fondos públicos de las CCAA analizadas, lo que evidencia la falta de estrategias que apoyen la intervención del profesorado, quedan expuesto a la improvisación sin una regulación que lo justifique, como indica Zych, Ortega-Ruiz \& Del Rey (2015).

Los resultados sobre el análisis del carácter de las medidas adoptadas hacia el agresor revelan que, en su mayoría, son correctivas y disciplinarias, lo cual queda lejos de las propuestas de la Convención de Naciones Unidas (2011b). Sobre las medidas con las víctimas, encontramos que no todas las normativas recogen actuaciones concretas, siendo de carácter protector. Es de señalar que en ningún caso hemos encontrado acciones preventivas y/o educativas, ni tampoco grupales (Cerezo, 2014; Caballo, et al., 2012).

Por otro lado, con este estudio se pone de relieve la actual preocupación normativa por controlar la violencia escolar, aunque no por su prevención (Ortega \& Núñez, 2012), ya que la mitad de las CCAA publicaron la normativa durante el período de tiempo comprendido entre los años 2009-2015, estando todavía en fase de formulación. Esto puede explicar la falta de conexión entre las políticas sobre acoso escolar, que aún se encuentran fragmentadas e inconsistentes (Comer \& Limber, 2015).

Entre las principales aportaciones del presente trabajo, destacar el hallazgo de que los protocolos que abordan la prevención, detección y actuación o intervención no son extensivos a todas las CCAA, siendo imprescindible como texto base a los centros escolares, que deberían reelaborarlo para adaptarlo a la realidad de sus aulas. Otro aspecto a destacar es la ausencia de respuestas educativas a las situaciones de bullying y cyberbullying, ya que casi exclusivamente son de carácter sancionador y disciplinario, presentando una contradicción con la naturaleza que deberían tener. Además, se evidencia la no inclusión de fenómenos de ciberacoso en la mayoría de legislaciones de las CC.AA., lo cual resulta incomprensible, dado que el uso de las TIC, como recurso, procedimiento y técnica de comunicación, forma parte de la cotidianidad de niños y adolescentes, por lo que es necesario abordar el cyberbullying en los códigos educativos. Finalmente, sorprende que en algunas autonomías no exista actualmente una norma específica para el acoso escolar o bullying.

Respecto a las limitaciones del estudio, la comparación de nuestro trabajo alcanzó los documentos normativos (leyes, decretos, órdenes y resoluciones) relativos al ámbito educativo y publicados por las comunidades autónomas seleccionadas (un total de ocho), prescindiendo de otras fuentes como los documentos institucionales (pactos sociales, planes y programas) relacionados con los fenómenos de acoso escolar y ciberacoso puesto que es posible que aunque en la legislación no se encuentre una clara respuesta al bullying y cyberbullying sí puede existir en otros documentos. 
En cuanto a las prospectivas futuras, consideramos que es necesario continuar en esta línea de investigación para conocer y comparar la legislación educativa relacionada con los fenómenos de acoso escolar y ciberacoso en el conjunto de las comunidades autónomas españolas, lo que aportaría información más precisa acerca del correcto o no abordaje de dichas realidades escolares presentes en los centros educativos de nuestro país.

\section{Referencias}

AA.VV. (2000). Violencia escolar: El maltrato entre iguales en la Educación Secundaria Obligatoria. Madrid, España: Informe de la Oficina del Defensor del Pueblo.

AA.VV. (2007). Violencia escolar: El maltrato entre iguales en la Educación Secundaria Obligatoria 1999-2006 (Nuevo estudio y actualización del informe 2000). Madrid, España: Informe de la Oficina del Defensor del Pueblo.

Álvarez-García, D., Núñez, C., Dobarro, A., \& Rodríguez, C. (2015). Risk factors associated with cybervictimization in adolescence. International Journal of Clinical and Health Psychology, 15, 226-235.

Benítez, J.L., Berbén, A.G., \& Fernández, M. (2006). El maltrato entre alumnos: conocimientos, percepciones y actitudes de los futuros docentes. Revista de Investigación Educativa, 24 (2), 329-352.

Bender, D. \& Lösel, F. (2011). Bullying at school as predictor of delinquency, violence and other anti-social behavior in adulthood. Criminal Behavior and Mental Health, 21(2), 99-106.

Bereday, G. (1964). Comparative Method in Education. New York, EEUU: Winston.

Boletín Oficial de Canarias (s.f.). (http://goo.gl/OepcGQ)

Boletín Oficial de Castilla y León (s.f.). (http://goo.gl/S01095)

Boletín Oficial de la Junta de Andalucía (s.f.). (http://goo.gl/A6YZuw)

Boletín Oficial de la Región de Murcia (s.f.). (http://goo.gl/nHwmeH)

Boletín Oficial de Madrid (s.f.). (https://goo.gl/mpuapy)

Boletín Oficial del Estado (s.f.). (http://goo.gl/LFrqhU)

Boletín Oficial del País Vasco (s.f.). (http://goo.gl/5pMa3R)

Caballo, V.E, Calderero, M., Arias, B., Salazar, I., \& Irurtia, M.J. (2012). Desarrollo y validación de una nueva medida de autoinforme para evaluar el acoso escolar (bullying). Psicología Conductual, 20(3), 625-647.

Calvete, E., Orue, I., Estévez, A., Villardón, L., \& Padilla, P. (2010). Cyberbullying in adolescents: Modalities and aggressors' profile. Computer in Human Behavior, 26, 1128-1135.

Cerezo, F. (2009a). La violencia en las aulas. Análisis y propuestas de intervención. Madrid, España: Pirámide.

Cerezo, F. (2009b). Bullying: análisis de la situación de las aulas españolas. International Journal of Psychology and Psychological Therapy, 9, 383-394. 
Cerezo, F. (2011). Políticas de convivencia escolar: percepción y eficacia desde la perspectiva familiar. Revista Electrónica Interuniversitaria de Formación del Profesorado (REIFOP), 14 (1), 313-323.

Cerezo, F. (2012). Bullying a través de las TIC. Boletín Científico Sapiens Research, 2(2), 24-29.

Cerezo, F. (2014). Diferencias en estatus social entre roles en bullying. Un análisis sociométrico. Bordón. Revista de Pedagogía, 66(2), 33-46.

Cerezo, F. (2015). Bullying homofóbico. El papel del profesorado. International Journal of Developmental and Educational Psychology, 1(1), 417-424.

Cerezo, F., Arnaiz, P., Giménez, A., \& Maquilón, J. (2016). Conductas de ciberadicción y experiencias de cyberbullying entre adolescentes de la Región de Murcia (España). Anales de Psicología, 32(3), 761,769.

Constitución Española de 1978 (artículos 15 y 27).

Comer, D. \& Limber, S. P. (2015). Law and Policy on the Concept of Bullying at School. American Psychological Association, 70 (4), 333-343.

Decreto 114/2011, de 11 de mayo, por el que se regula la convivencia en el ámbito educativo de la Comunidad Autónoma de Canarias.

Decreto 15/2007, de 19 de abril, por el que se establece el marco regulador de la convivencia en los centros docentes de la Comunidad de Madrid.

Decreto 19/2007, de 23 de enero, por el que se adoptan medidas para la promoción de la Cultura de Paz y Mejora de la Convivencia en los Centros Educativos sostenidos con fondos públicos en la Comunidad Autónoma de Andalucía.

Decreto 201/2008, de 2 de diciembre, sobre los derechos y deberes de los alumnos y alumnas de los centros docentes no universitarios de la Comunidad Autónoma del País Vasco.

Decreto 279/2006, de 4 de julio, sobre los derechos y deberes del alumnado y regulación de la convivencia en los centros educativos no universitarios de Cataluña.

Decreto 51/2007, de 17 de mayo, por el que se regulan los derechos y deberes de los alumnos y la participación y los compromisos de las familias en el proceso educativo, y se establecen las normas de convivencia y disciplina en los centros educativos de Castilla y León.

Decreto $8 / 2015$, de 8 de enero, por el que se desarrolla la Ley 4/2011, de 30 de junio, de convivencia y participación de la comunidad educativa en materia de convivencia escolar en la Comunidad Autónoma de Galicia.

Decreto n. ${ }^{\circ} 119 / 2005$, de 21 de octubre, por el que se establecen las normas de convivencia en los centros docentes sostenidos con fondos públicos que imparten enseñanzas escolares en la Comunidad Autónoma de la Región de Murcia.

Diari Oficial de la Generalitat de Catalunya (s.f.). (http://goo.gl/7C8tTi)

Diario Oficial de Galicia (s.f.). (http://goo.gl/MBwKgn)

Durán-Segura, M. \& Martínez-Pecino, R. (2015). Ciberacoso mediante teléfono móvil e internet en las relaciones de noviazgo entre jóvenes. Comunicar, 44 (22), 156-167.

Fanjul, J.M. (2012). Visión jurídica del acoso escolar (Bullying). Avances en supervisión educativa. Revista de la Asociación de Inspectores de Educación de España, 17, 1-8. 
Fernández-Montalvo, J. \& Peñalva, A. (2015). Hábitos de uso y conducta de riesgo en Internet en la preadolescencia. Comunicar, 44(22), 113-120.

Fiscalía General del Estado. Instrucción 10/2005, Sobre el tratamiento del acoso escolar desde el sistema de Justicia juvenil.

Garaigordobil, M. (2011). Prevalencia y consecuencias del cyberbullying: una revisión. International Journal of Psychology and Psychological Therapy, 11(2), 233-254.

Garaigordobil, M. \& Aliri, J. (2013). Ciberacoso ("Cyberbullying”) en el País Vasco. Diferencias de sexo en víctima, agresores y observadores. Psicología Conductual, 21(3), 461-474.

García-Bacete, F. J., Sureda, I., \& Monjas, M. I. (2010). El rechazo entre iguales en la educación primaria: una panorámica general. Anales de Psicología, 26(1), 123-136.

Horner, S., Asher, Y., \& Fireman, G.D. (2015). The impact and response to electronic bullying and traditional bullying among adolescents. Computers in Human Behavior, 49, 288295.

Instrucción 10/2005 de 6 de octubre, sobre el tratamiento del acoso escolar desde el sistema de Justicia Juvenil.

Ley 12/2009, de 10 de julio, de Educación de Cataluña.

Ley 4/2011, de 30 de junio, de convivencia y participación de la comunidad educativa en la Comunidad Autónoma de Galicia.

Ley Orgánica 10/1995, de 23 de noviembre, del Código Penal.

Ley Orgánica 5/2000, de 12 de enero, reguladora de la responsabilidad penal de los menores.

Ley Orgánica 8/2006, de 4 de diciembre, por la que se modifica la Ley Orgánica 5/2000, de 12 de enero, reguladora de la responsabilidad penal de los menores.

Ley Orgánica de Educación 2/ 2006.

López, L. \& Ramírez, A. (2014). Medidas disciplinarias en los centros educativos: ¿suficientes contra el acoso escolar? Perfiles Educativos, 36(145), 32-50.

Moreno, D., Estévez, E., Murgui, S., \& Musitu, G. (2009). Relación entre el clima familiar y el clima escolar: el rol de la empatía, la actitud hacia la autoridad y la conducta violenta en los adolescentes. International Journal of Psychology and Psychological Therapy, 9(1), 123-136.

Musitu, G., Estévez, E., Jiménez, T., \&Veiga, F. H. (2011). Agentes de socialização da volência e vitimização escolar].En S. N. Caldeira, F. H. Veiga (dir.), Interviremsituações de indisciplinaviolência e conflito (pp. 43-80). Lisboa, Portugal: Fim de Século.

Naciones Unidas (1989) Comité de los Derechos del Niño. Artículo 19 de la Convención de Naciones Unidas sobre los Derechos del Niño.

Naciones Unidas (2011a). Comité de los Derechos Económicos, Sociales y Culturales. Observación General №13. El Derecho a la Educación.

Naciones Unidas (2011b). Comité de los Derechos del Niño. Observación General №13. Derecho del niño a no ser objeto de ninguna forma de violencia.

Olweus, D. (1996). Bullying and school. Oxford, Inglaterra: Blackwell.

Olweus, D. (1998). Conductas de acoso y amenaza entre escolares. Madrid, España: Morata. 
Orden de 20 de junio de 2011, por la que se adoptan medidas para la promoción de la convivencia en los centros docentes sostenidos con fondos públicos y se regula el derecho de las familias a participar en el proceso educativo de sus hijos e hijas en la Comunidad Autónoma de Andalucía.

Orden EDU/1921/2007, de 27 de noviembre, por la que se establecen medidas y actuaciones para la promoción y mejora de la convivencia en los centros educativos de Castilla y León.

Orden EDU/52/2005, de 26 de enero, relativa al fomento de la convivencia en los centros docentes de Castilla y León.

Ortega, R. (2008). Malos tratos entre escolares. De la investigación a la intervención. Madrid, España: CIDE-Secretaría General Técnica.

Ortega, R. \& Núñez, J. C. (2012). Bullying and cyberbullying: Research and intervention at school and social contexts. Psicothema, 24(4), 603-607.

Ortega, R., Sánchez, V., \& Menesini, E. (2002). Violencia entre iguales y desconexión moral: un análisis transcultural. Psicothema, 14, 37-49.

Preámbulo de la Ley de Ordenación General del Sistema Educativo (LOGSE, 1990).

Prodócimo, E., Cerezo, F., \& Arense, J.J. (2014). Acoso escolar: variables sociofamiliares como factores de riesgo o de protección. Psicología Conductual, 22(2), 345-359.

Real Decreto 732 / 1995, de 5 de mayo.

Resolución de 28 de septiembre de 2009, de la Dirección General de Ordenación Académica, sobre aspectos relativos a la aplicación de las normas de convivencia en la Comunidad Autónoma de la Región de Murcia.

Resolución de 4 de abril de 2006, de la dirección general de ordenación académica, por la que se dictan instrucciones en relación a situaciones de acoso escolar en los centros docentes sostenidos con fondos públicos que imparten enseñanzas escolares en la Comunidad Autónoma de la Región de Murcia.

Sánchez-Meca, J. \& Botella, J. (2010). Revisiones sistemáticas y meta-análisis: herramientas para la práctica profesional. Papeles del Psicólogo, 31(1), 7-17.

Save the Children (2016). Acoso escolar y ciberacoso: propuestas para la acción. Madrid, España: Save the Children

Smith, P.K., Mahdavi, J., Carvalho, M., Fisher, S., Russel, S., \& Tippet, N. (2008). Cyberbullying: its nature and impact in secondary school pupils. Journal of Child Psychology and Psychiatry, 49(4), 376-385.

Solberg, M. \& Olweus, D. (2003). Prevalence estimation of school bullying with the Olweus Bully/Victim Questionnaire. Aggressive Behavior, 29, 239-268.

Viana-Orta, M.I. (2013). La mediación escolar en los planes y programas institucionales de convivencia en España. Revista Complutense de Educación, 25(2), 271-291.

Zych, I., Ortega-Ruiz, R., \& Del Rey, R. (2015). Systematic review of theoretical studies on bullying and cyberbullying: Facts, knowledge, prevention, and intervention. Aggression and Violent Behavior, 23, 1-21. 